version 1.0

\title{
Wetting phenomenon in the liquid-vapor phase coexistence of a partially miscible Lennard-Jones binary mixtures
}

\author{
Enrique Díaz-Herrera ${ }^{a}$, José A. Moreno-Razo $^{a}$ and Guillermo Ramírez-Santiago ${ }^{b}$ \\ ${ }^{a}$ Departamento de Física, Universidad Autónoma Metropolitana-Iztapalapa, \\ Apdo. Postal 55-534, México 09340, D.F., MEXICO \\ ${ }^{b}$ Instituto de Física, Universidad Nacional Autónoma de México, \\ Apdo. Postal 20-364 México 01000, D. F. MEXICO
}

\begin{abstract}
We have carried out extensive equilibrium molecular dynamics (MD) simulations to study the structure and the interfacial properties in the liquid-vapor (LV) phase coexistence of partially miscible binary Lennard-Jones (LJ) mixtures. By analyzing the structural properties as a function of the miscibility parameter, $\alpha$, we found that at relatively low temperatures the system separates forming a liquid A-liquid B interface in coexistence with the vapor phase. At higher temperatures and, $0<\alpha \leq 0.5$, we found a temperature range, $T_{w}^{*}(\alpha) \leq T^{*}<T_{\text {cons }}^{*}(\alpha)$, where the liquid phases are wet by the vapor phase. Here, $T_{w}^{*}(\alpha)$ represents the wetting transition temperature (WTT) and $T_{\text {Cons }}^{*}(\alpha)$ is the consolute temperature of the mixture. However, for $0.5<\alpha<1$, no wetting phenomenon occurs. For the particular value, $\alpha=0.25$, we analyzed quantitatively the $T^{*}$ versus $\rho^{*}$, and $P^{*}$ versus $T^{*}$ phase diagrams and found, $T_{c}^{*} \simeq 1.25$, and $T_{\text {cons }}^{*} \simeq 1.25$. We also studied quantitatively, as a function of temperature, the surface tension and the adsorption of molecules at the liquid-liquid interface. It was found that the adsorption shows a jump from a finite negative value up to minus infinity, when the vapor wets the liquid phases, suggesting that the wetting transition (WT) is of first order. The calculated phase diagram together with the wetting phenomenon strongly suggest the existence of a tricritical point. These results agree well with some experiments carried out in fluid binary mixtures.
\end{abstract}

PACS numbers: 68.03.Cd 68.03.Hj 68.05.Cf 68.08.Bc 


\section{INTRODUCTION}

Wetting phenomena occurs very often in daily life and has a strong interdisciplinary character. It is of great relevance for fundamental areas of knowledge such as physics [1, 2], chemistry [3], biology 4], and several other applied sciences [5] as well as technology [6]. The occurrence of wetting phenomenon is usually associated with the existence of three phases, at least one of which is liquid and no more than one phase is solid. In most practical situations the solid phase is wet by the liquid phase and the disfavored phase is the vapor. This is expressed in terms of surface free energies as, $\gamma_{S L}<\gamma_{S V}$. Although the vapor phase is disfavored, a solid-vapor coexistence can be possible and in such case it is said that the liquid "partially wets" the solid. However, as the liquid phase is further adsorbed by the solid, it is possible that the contact of the vapor with the solid be excluded by the creation of a liquid layer between them. In such a situation one says that the liquid "completely wets" the solid. The surface free energies involved in the wetting process are related by Young's rule, $\gamma_{S V}=\gamma_{S L}+\gamma_{L V} \cos \theta$, with $\theta$ the contact angle. For partial wetting, $0<\theta<90^{0}$, and for complete wetting, $\theta=0$. Usually the presence of a wall in most of the theoretical as well as experimental studies complicates a detailed and precise investigation of the interfacial structures. This is due to the fact that the atomic interactions between the wall and the fluid components play an important role in the wetting phenomena. Fortunately, binary liquid mixtures offer a great opportunity to investigate in detail wetting phenomena since they involve only fluid phases in coexistence. An important number of experimental studies of interfacial wetting have studied only a small subset of possible binary mixtures [1, 1, 8, 9 , 10, 11]. Wetting and prewetting phenomena in which one of the phases is solid has also been studied by means of Monte Carlo simulations using a 3D lattice-gas model [12]. A 12-6 LJ potential modeling argon and a 9-3 LJ potential modeling a $\mathrm{CO}_{2}$ covered solid wall have been chosen to study the wetting transitions by means of Monte Carlo simulations [13]. However, to the best of our knowledge, there are not numerical simulations that explicitly study the interfacial behavior and the associated wetting phenomena in fluid mixtures in absence of a wall. In this paper we apply a well established methodology using MD simulations 14, 15, 16, 17, 18] to study interfacial properties and surface phenomena in a partially miscible fluid mixture. We consider a model binary LJ fluid mixture in which the attractive part of the A-B interactions is weaker than the A-A and B-B interactions. By studying the properties of the density profiles of the fluid phases as a function of temperature and miscibility parameter, 
we show clear evidence that the vapor phase spontaneously excludes the liquid A-liquid B interface at and above the wetting transition temperature, $T_{\mathrm{W}}^{*}$. That is, the vapor phase fully wets the liquid A-liquid B phases. This phenomenon occurs when the miscibility parameter $\alpha$ is in the range $0<\alpha \leq 0.5$ and in the temperature region $T_{W}^{*}(\alpha)<T^{*}<T_{\text {Cons }}^{*}(\alpha)$. Here, $T^{*}=\left(k_{B} T\right) / \epsilon$, is the reduced temperature, $T_{\mathrm{w}}^{*}(\alpha)$, is the wetting transition temperature (WTT), and $T_{\mathrm{Cons}}^{*}(\alpha)$, is the consolute temperature of the mixture. By analyzing the structural properties of the mixture as a function of $\alpha$ we estimate the wetting phase diagram $T_{\mathrm{w}}^{*}(\alpha)$. A further quantitative analysis, for $\alpha=0.25$, of the surface free energy $\gamma\left(T^{*}\right)$ and the adsorption, $\Gamma\left(T^{*}\right)$, at the LL interface indicates that the wetting transition is of first order.

The layout of the remainder of this paper is as follows. In section [1] we introduce the model of the symmetric binary mixture, in section [II we explain the details of the simulations, then in section IV we present and discuss the representative results of the extensive MD simulations. Finally, we end with the conclusions in section $\mathrm{V}$

\section{THE MODEL}

The model binary mixture studied in this paper consists of fluids A and B made up of spherical molecules of the same size, $\sigma_{A A}=\sigma_{B B}$, and at concentrations of $50 \%$ each. The interaction between molecules of the same type is through the classical 12-6 LJ potential. However, the interaction between molecules of fluids A and B is given by the LJ potential,

$$
u_{i j}(r)= \begin{cases}4 \epsilon_{i j}\left[\left(\frac{\sigma_{i j}}{r_{i j}}\right)^{12}-\left(\frac{\sigma_{i j}}{r_{i j}}\right)^{6}\right], & \text { if } r \leq R_{c}=3 \sigma_{A A}, \\ 0, & \text { if } r>R_{c}=3 \sigma_{A A},\end{cases}
$$

where the mixing rule is defined by

$$
\begin{aligned}
\sigma_{A B} & =\frac{1}{2}\left(\sigma_{A A}+\sigma_{B B}\right), \\
\epsilon_{A B} & =\alpha_{A B} \epsilon_{A A},
\end{aligned}
$$

with $\epsilon_{A A}=\epsilon_{B B}$, and $\alpha_{A B}$ the parameter that controls the miscibility of the two fluids. For the sake of simplicity from now on we will use the short notation, $\alpha_{A B}=\alpha$. Notice that when, $\alpha=0$, we obtain two independent single LJ fluids while in the opposite case, $\alpha=1$, the system reduces to a single LJ fluid. By choosing, $0<\alpha<1$, the attractive part of the A-B interactions becomes weaker than that of the AA and BB interactions, and then, 
the liquid phases are immiscible in a wide range of temperatures. Thus, one can obtain the coexistence of three fluid phases: liquid A-liquid B and the vapor.

\section{DETAILS OF THE SIMULATIONS}

We have carried out extensive MD simulations to investigate the structural properties of this model binary mixture as a function of $\alpha$, varying this quantity in steps, $\delta \alpha=0.05$, in the interval $0.2 \leq \alpha \leq 0.5$. For $\alpha=0.25$ we quantitatively related the interfacial properties with the corresponding phase diagram properties. In all the simulations we applied periodic boundary conditions along the $x, y$ and $z$ directions. At the lowest temperature, $T^{*}=0.65$, the simulations were initiated from a configuration where the molecules of type A and B form two contiguous FCC crystals. At higher temperatures we take as the initial configuration the final configuration of the previous temperature. The initial velocities of the molecules were assigned from a Maxwell-Boltzmann distribution. The equations of motion were integrated using a leap frog algorithm with a time step size, $\delta t^{*}=0.005$. This corresponds to $1.1 \times 10^{-5}$ nanoseconds in the scale of argon. At each time step iteration we monitor the temperature of the system, by means of the equipartition theorem, and rescale the linear momentum of the molecules to keep the temperature constant. This method of rescaling the linear momenta is known as the isokinetic thermostat. To check that this isokinetic thermostat produces meaningful results we have also carried out some MD simulations using the NoséHoover thermostat. In figure 1 we show the kinetic energy distributions as the dynamics of the system evolves at, $T^{*}=0.90$, applying both methods. As one would expect both thermostats yield a Gaussian distribution of kinetic energies. The only difference is the width of the distributions. The isokinetic thermostat produced a distribution with a variance that is one order of magnitude smaller than the variance of the distribution obtained with the Nosé-Hoover thermostat. As a further check of the isokinetic thermostat we also calculated and monitored some thermodynamic and surface quantities applying both thermostats. The results of this comparison test showed full consistency. Since MD simulations using the NoséHoover thermostat are computationally more demanding we used the isokinetic thermostat

in all the simulations reported in this paper. Furthermore, to check the stability of the interfaces as well as the distribution of the species, we simulated the system for as long as $55 \mathrm{~ns}$ in the scale of Argon. Thermodynamic quantities and interfacial properties of interest were measured by averaging over the last million of time-step iterations. To minimize 
correlations between measurements we calculated thermodynamic, structural and surface quantities every 50 time steps. We also investigated the role of finite size effects for the value of the miscibility parameter, $\alpha=0.25$. To this end we carried out MD simulations with three system sizes, $\mathrm{N}=1728,4096$ and 6144 molecules. We found that for all the quantities studied here, simulations with $N=4096$ provided reliable results. Therefore, most of the simulations were carried out with $N=4096$ molecules. The discussion of these results will be presented, where appropriate, in the next section. On the other hand, interfacial properties are sensitive to the cross section area of the simulational box that is parallel to the interfaces, as discussed in previous MD simulations of the LV interface of a single LJ fluid [18, 19]. These authors arrived to the conclusion that a reliable value of the cross section area of the computational box should be at least $(8 \sigma)^{2}$. So, to be on the safe side, in the present simulations we have considered a computational box with a cross section area, $L x \times L y=\left(9 \sigma_{A A}\right)^{2}$. The length, $L_{z}$, of the simulational parallelepiped was adjusted such that the average density of the system laid somewhere inside the LV coexistence curve. In this way one readily gets the liquid-vapor phase coexistence. The average densities of the simulated systems were in the range $0.2 \leq \rho^{*} \leq 0.4$, where the reduced density defined as, $\rho^{*}=\rho \sigma_{\mathrm{AA}}^{3}$. In the following section we present, analyze and discuss the results of the thermodynamic and interfacial properties calculated from our MD simulations.

\section{RESULTS AND DISCUSSION}

\section{A. Structural properties and phase diagrams}

We performed extensive MD simulations for mixtures with $0.2 \leq \alpha \leq 0.65$ changing this parameter in steps of, $\delta \alpha=0.05$. We studied the density profiles, $\rho^{*}(z)$, of the liquidvapor coexistence in different temperature regions. From an analysis of $\rho^{*}(z)$, as function of $T^{*}$, and, $\alpha$, we estimated the $T_{\mathrm{W}}$ versus $\alpha$ phase diagram. In what follows we present some representative results for, $\rho^{*}(z)$, when $\alpha=0.25$ and 0.30 . Then, for the particular value, $\alpha=0.25$, we present a quantitative analysis of the $T^{*}$ versus $\rho^{*}$, and $T^{*}$ versus $P^{*}$ phase diagrams. We also give a brief description of the procedure we followed to locate the coexistence courve and the $-\lambda$ line- mixing-demixing line.

Once the system reached equilibrium we calculated the structural properties of the system from simulations with $N=4096$ molecules. In figure 2 we show the density profiles at the 
relatively low temperatures, $T^{*}=0.65$ and 0.75 , when $\alpha=0.25$. In this temperature region the liquid-vapor equilibrium structure of the mixture consists of a liquid A-liquid B interface in coexistence with the vapor phase. As temperature increases and reaches the region $0.80 \leq T^{*} \leq 1.25$, however, this fluid phase structure rearranges in such a way that, the vapor phase spontaneously sets in between the Liquid A-Liquid B phases, as shown in figure 3. This structure remains stable during all the time of the simulation, about $55 \mathrm{~ns}$ in the scale of argon. This is a clear evidence that the vapor phase wets the liquid phases. In figure 4 we show similar results for $\alpha=0.3$. There one sees that at, $T^{*}=0.82$, the structure of the system is such that there is a liquid A-liquid B interface. Nonetheless, at higher temperatures, for instance, $T^{*}=0.9$, the vapor phase wets the liquid phases. This behavior of $\rho^{*}(z)$ for $\alpha=0.3$, suggest that $T_{\mathrm{W}}^{*}(\alpha=0.25)<T_{\mathrm{W}}^{*}(\alpha=0.3)$. In fact, comparing the structures plotted in figures 3 and 4 one should note that the mixture with $\alpha=0.25$ already wets at, $T^{*}=0.83$. Following a systematic analysis of the structure of density profiles as function of temperatures for all the values of $\alpha$ we estimated the wetting transition temperatures $T_{\mathrm{W}}^{*}(\alpha)$. We found that $T_{\mathrm{W}}^{*}$ increases monotonically as a function of $\alpha$, whenever $0<\alpha \leq 0.5$. For higher values, $0.5<\alpha<1.0$, this wetting phenomenon does not occur. The results are summarized in the wetting phase diagram in figure 5 We believe that the reason for which the system wets below $\alpha=0.5$, and no longer does above this value is due to the equal size of the molecules of type $\mathrm{A}$ and $\mathrm{B}$ and because $\varepsilon_{A A}=\varepsilon_{B B}$.

Now we will try to relate these density profiles structure and wetting phenomenon with the properties of the phase diagram of the mixture. To this end we will discuss in detail the properties of the corresponding phase diagram for $\alpha=0.25$. To begin it is important to remind that the present model binary mixture corresponds to the type III in the classification of Scott and Konynenburg [20]. The phase diagram properties of this kind of mixtures has been quantitatively studied by Wilding et. al. [21], using a square well potential for the intermolecular interactions. They showed that for a strong immiscible binary mixture a tricritical point exists. This means that the $\lambda$ line meets the LV coexistence curve at the critical point. In figure 6 we show the $T^{*}$ versus $\rho^{*}$ phase diagram of the mixture obtained from extensive MD simulations with $\alpha=0.25$. Since for $\alpha=0$ and 1 our model reduces to single LJ fluids, we have also included in the same figure, for comparison, the phase diagram of a single LJ fluid. Both phase diagrams were calculated simulating a system with $N=4096$ molecules and using a shifted intermolecular potential with a cutoff of $3 \sigma$. Therefore, the LV critical temperature of the single fluid became $T_{C}^{L J} \simeq 1.2$. One should note that, the mixture 
critical density is higher than that of the single LJ fluid, and its critical temperature shifts upwards. This shift in $\rho_{c}^{*}$ and $T_{c}^{*}$ occurs due to the fact that the less miscible the mixture is, smaller $\alpha^{-}$, the larger the temperature range of immiscibility. In addition, the $\lambda$ line appears to touch the LV critical point, and the critical point becomes tricritical. These results agree well with those obtained recently for square well strong immiscible binary mixtures 21].

To check how sensitive are the phase diagram properties of the mixture, for $\alpha=0.25$, to the number of molecules in the simulations, we also calculated the phase boundaries simulating a system with $N=1728$ molecules. In fig. 7 we show the results of this finite size analysis. For a mixture with $N=1728$ the density of the liquid phase decreases while the density of the vapor phase increases. We also observe that at low temperatures the results are system size independent. However, as temperature increases, in particular, close to the critical point, there are differences in the phase boundaries obtained using $N=1728$ and 4096 molecules. As one approaches the critical point it turns out more difficult to determine the coexistence densities with a system with $N=1728$ molecules. This is so because the difference in the coexistence densities becomes smaller, and the number of particles in the system is not sufficient to give rise to bulk fluid phases. Nevertheless, for a system with $N=4096$ this is not the case and we indeed obtained the liquid and vapor fluid phases. On the other hand, it is known that near to the critical point the fluctuations of the density are strong and the vapor and liquid densities are not well defined. This fact complicates the location of the critical point. To circumvent this difficulty we proceed as in reference [22] and calculated the total density distributions in a system with $N=4096$, at several temperatures around $T_{c}$. The simulational box was divided in several slabs, parallel to the interface, of width between $\sigma$ and $3 \sigma$. The density of particles was calculated in each slab every 50 time steps of the MD simulations. A block average histogram of the densities is obtained every $5 \times 10^{4}$ time steps. The resulting total density distribution, $\rho_{A}+\rho_{B}$, was calculated after averaging over 20 blocks. The result is presented in figure 8 . At temperatures slightly below $T_{c}$, we obtained density distributions that show two maxima. The low density maximum corresponds to the vapor phase and the higher maximum corresponds to the liquid density. Nonetheless, at temperatures above $T_{c}$, the density distributions show only one maximum. In Fig 8 we show the total density distributions at $T^{*}=1.1,1.15$ and 1.2. The low density maximum is higher because the volume of the vapor phase is larger than the volume of the liquid phases. Thus, the vapor density appears with a higher frequency in the histograms.

To locate of mixing-demixing transition temperatures, $-\lambda$ line- for $\alpha=0.25$ and $N=$ 
4096, we followed a similar procedure as that described for the location of the LV critical point. However, in the determination of the $\lambda$ line, we only consider the density distribution of one of the species, $\rho_{A}$ or $\rho_{B}$. The reason is that when the system is in the demixing region, the density distribution of one of the species shows two maxima. The low density peak corresponds to the poor fluid phase and the high density peak correspond to the rich fluid phase. Nonetheless, when the system is in the mixing region the fluid phases become homogeneous and therefore one should observe only one peak in the density distribution. Therefore, to locate the mixing-demixing points we looked at the transition from the two peak structure density distribution to one peak density distribution. This analysis was done as a function of the total density of the system and at three different temperatures. The results are shown in figure 9]

Another way of locating the $\lambda$ line is calculating the pressure versus temperature phase diagram, shown in figure 10, for a system with $N=4096$ molecules. The pressure was calculated as the average of the pressure tensor component perpendicular to the interface via the virial formula [23]. There we also included for comparison, the results for a single LJ fluid with the same number of molecules. We found that the LV phase boundary is located right at $T^{*} \simeq 1.25$. At higher temperatures there are two branches that were obtained by approaching the mixing-demixing boundary from both sides of the $\lambda$ line. These branches enclose a region that is narrower in size, as compared to the region obtained in the $\rho^{*}$ versus $T^{*}$ phase diagram. These results suggest that the calculation of the $P^{*}$ versus $T^{*}$ phase diagram gives more precise way to locate the mixing-demixing line. Again, we did find evidence that suggest that the $\lambda$ line meets the LV coexistence line at the LV critical point. These results give a strong support to the existence of a tricritical point [21].

\section{B. Interfacial properties}

Now we turn to the discussion of the interfacial properties and surface phenomenon of the mixture at the liquid-vapor phase coexistence. We carried out a quantitative analysis of these properties for $\alpha=0.25$. To evaluate the wetting transition temperature with precision we calculated the interfacial free energies as a function of temperature. To this purpose we use the well known formula,

$$
\gamma=\int_{\text {bulk }_{1}}^{\text {bulk }_{2}}\left(P_{n}(z)-P_{t}(z)\right) d z
$$


where the integrations were carried out up to the middle of the corresponding bulk phases. The tangential and normal pressure profiles were calculated using the definition of the IrvingKirkwood pressure tensor [23]. For a planar interface these pressure profiles are given by the formula [23, 24].

$$
\begin{aligned}
P_{n}(z) & =\rho(z) k_{B} T \\
& -\frac{1}{2 A}\left\langle\sum_{i \neq j} \frac{z_{i j}^{2} u_{i j}^{\prime}\left(r_{i j}\right)}{r_{i j}\left|z_{i j}\right|} \theta\left(\frac{z-z_{i}}{z_{i j}}\right) \theta\left(\frac{z_{j}-z}{z_{i j}}\right)\right\rangle, \\
P_{t}(z)= & \rho(z) k_{B} T \\
& -\frac{1}{4 A}\left\langle\sum_{i \neq j} \frac{\left[x_{i j}^{2}+y_{i j}^{2}\right] u_{i j}^{\prime}\left(r_{i j}\right)}{r_{i j}\left|z_{i j}\right|} \theta\left(\frac{z-z_{i}}{z_{i j}}\right) \theta\left(\frac{z_{j}-z}{z_{i j}}\right)\right\rangle .
\end{aligned}
$$

According to Young's rule the difference,

$$
\Delta(T)=2 \gamma_{\mathrm{LV}}-\gamma_{\mathrm{LL}}
$$

must be zero at the WTT. So, the wetting by the vapor phase occurs when the free energy difference, $\Delta$, becomes negative. This quantity is plotted as a function of reduced temperature in the inset of figure 11 for $\alpha=0.25$ and $N=4096$. A linear extrapolation of the data indicates that the wetting occurs at about $T_{\mathrm{W}}^{*}(\alpha=0.25)=0.80$. Notice that due to the planar geometry of the interfaces there is no contact angle and the three surface tensions are independent and considered separately. To improve the accuracy of $T_{\mathrm{W}}^{*}(\alpha=0.25)$, one needs to carry out even more demanding simulations. This is due to the fact that the interfacial tension always shows relatively large fluctuations. The situation complicates even more when the simulations are performed at temperatures very close to the WTT. A second independent way to estimate the WTT and to figure out the nature of the WT, we calculated the adsorption of molecules at the LL interfaces shown in figures 2 . This is done using the formula,

$$
\Gamma=\int_{\text {bulkA }}^{\text {bulkB }}\left(\rho(z)-\rho_{\text {bulk }}\right) d z,
$$

The results of the calculations for $\alpha=0.25$ and $N=4096$ and 6148 are plotted in figure 12 . One sees that $\Gamma\left(T^{*}, \alpha=0.25\right)$ decreases monotonically in the temperature range, $0.75 \leq$ $T^{*} \leq 0.78$, and it consistently shows negative values. This is due to the inhomogeneity at the LL interface, since the density there, is much smaller than the density of the liquid bulk phases. Nonetheless, as $T^{*} \rightarrow 0.80$ from below the adsorption jumps from a finite negative value up to minus infinite since the vapor wets the LL interface at $T^{*}(\alpha=0.25) \simeq 0.80$. Note 
that the WTT shifts slightly towards higher temperatures as the number of molecules in the system increases from $N=4096$ up to $N=6148$. This jump or discontinuity is a strong indication that the WT is of first order. As expected the closer the temperature approach to $T_{W}^{*}(\alpha)$ the stronger the fluctuations in $\Gamma\left(T^{*}, \alpha\right)$. In figure 12 the closest approach to the

WTT, in reduced temperature, was $\frac{\delta T}{T_{W}}=6.25 \times 10^{-3}$. One would expect that the wetting transition is of first order even for other values of the miscibility parameter, $0<\alpha \leq 0.5$. This is so, since the behavior of $\rho^{*}(z, \alpha)$ is similar to that of $\alpha=0.25$, whose surface properties were studied in detail.

\section{CONCLUSIONS}

We carried out extensive MD simulations to study the LV phase coexistence, the structural properties and interfacial phenomena of a partially miscible symmetrical LJ binary mixture. By analyzing the density profiles as a function of temperature and miscibility parameter we estimated the wetting phase diagram, $T_{\mathrm{W}}^{*}$ versus $\alpha$. The wetting of the vapor phase happens whenever $0<\alpha \leq 0.5$. We also found that $T_{\mathrm{W}}^{*}(\alpha)$, monotonically increases up to $\alpha=0.5$. For other values of $\alpha$, this wetting phenomenon does not occur. In addition, we also studied quantitatively the $T^{*}$ versus $\rho^{*}$ and $P^{*}$ versus $T^{*}$ phase diagrams for $\alpha=0.25$. The results indicate that the former phase diagram shows a similar topology as that obtained for a square-well potential mixture estimated by means of mean-field theory and Monte Carlo simulations [21]. An analysis of the behavior of the adsorption of particles, at the LL interface, as a function of $T^{*}$, for $\alpha=0.25$, led to the conclusion that the WT is of first order. These results should be valid for a family of mixtures of the type III, in the classification of Scott and Konynemburg. The phase diagrams discussed here together with the wetting phenomenon are an explicit quantitative demonstration of the scenario suggested some time ago based on a microscopic expression for the Hamaker constant [25]. To the best of our knowledge this is the first time that this wetting phenomenon is quantitatively studied by means of MD simulations in binary LJ fluid mixtures in absence of a wall.. We would like to point out that this wetting phenomena agrees well with some experimental studies carried out with fluid binary mixtures. Finally, the results reported in this paper provide with a more complete understanding of the surface phenomena in partially miscible fluid binary mixtures. 


\section{ACKNOWLEDGMENTS}

EDH would like to thank the DAAD-Germany for financial support during the summer of 1999. Partial support from CONACYT contracts L0080-E9607 (EDH) and 25298-E (GRS) as well as from DGAPA-UNAM contracts IN110103, and IX107704, is also acknowledge.

[1] V. F. Kozhevnikov, D. J. Arnold, S. P. Naurzakov and M. E. Fisher, Phys. Rev. Lett. 78, 1735 (1997).

[2] W. K. Wijting, N.A. M. Besseling, and M. A. Cohen Stuart, Phys. Rev. Lett. 90, 196101 (2003).

[3] D. Ross, et. al. Phys. Rev. Lett. 87, 176103, (2001).

[4] R. Piazza, M. Pierno and E. Vignati, Phys. Rev. Lett. 90, 208101, (2003).

[5] Y. Q. Sun and T. Gao, Metallurgical and Materials Trans. 33A, 3285, (2002).

[6] M. L. Gutierrez and Kraemer D. Lukas, Jour. Chem. Eng. Data, 46, 679 (2001); Graue A., Bogno T., Baldwin T. A., S. P. E. Reservoir Evaluation and Engineering, 4, 455 (2001).

[7] O’D Kwon, D. Beaglehole, W. Webb and B. Widom, Phys. Rev. Lett. 48, 185 (1982).

[8] C. Houessou, P. Guenoun, R. Gastaud, F. Perrot and D. Beysens, Phys. Rev. A 32, 1818 (1985).

[9] M. Kahlweit, G. Busse, D. Hasse and J. Jeu, Phys. Rev. A 38, 1395 (1988).

[10] D. Bonn, H. Kellay and G. H. Wegdam, Phys. Rev. Lett. 69, 1975 (1992).

[11] H. Kellay, D. Bons, and J. Meunier, Phys. Rev. Lett. 71, 2607 (1993).

[12] D. Nicolaides and R. Evans, Phys. Rev. Lett. 63, 778 (1989); ibid Phys. Rev. A 39, 9336 (1989).

[13] J. E. Finn and P. A. Monson, Phys. Rev. A 39, 6402 (1989).

[14] G. A. Chapela, G. Saville, and J. S. Rowlinson, Faraday Discuss. Chem. Soc. 59, 22 (1975); G. A. Chapela, G. Saville, S. M. Thompson, and J. S. Rowlinson, J. Chem. Soc. Faraday Trans. 2731133 (1977).

[15] M. Mecke, J. Winkelmann, and J. Fischer, J. Chem. Phys. 107, 9264 (1997); Chem. Phys. 110, 1188 (1999).

[16] E. Díaz-Herrera, J. Alejandre, G. Ramírez-Santiago, and F. Forstmann, Chem. Phys. 110, 8084 (1999); E. Díaz-Herrera, G. Ramírez-Santiago, and J. A. Moreno-Razo, Phys. Rev. E 
68, 61204 (2003).

[17] M. Gónzalez-Melchor, J. Alejandre, and F. Bresme, Phys. Rev. Lett. 90, 135506 (2003).

[18] B. D. Holcomb, P. Clancy, and J. A. Zollweg, Mol. Phys. 78, 437 (1993).

[19] Li-Jen Chen, J. Chem. Phys. 103, 10214 (1995).

[20] R. L. Scott and P. H. van Konynenburg, Discuss. Faraday Soc. 49, 87 (1970).

[21] N. B. Wilding, F. Schmid and P. Nielaba, Phys. Rev. E 58, 2201, (1998).

[22] K. Binder editor, Topics in Applied Physics, Vol. 71. The Monte Carlo Methods in Condensed Matter Physics. (Springer Verlang, 1992).

[23] J.S. Rowlinson and B. Widom, Molecular Theory of Capillarity, (Clarendon Oxford, 1982).

[24] M. Rao and B. Berne, Mol. Phys. 37, 455 (1979).

[25] T. Getta and S. Dietrich, Phys. Rev. E 47, 1856 (1993). 


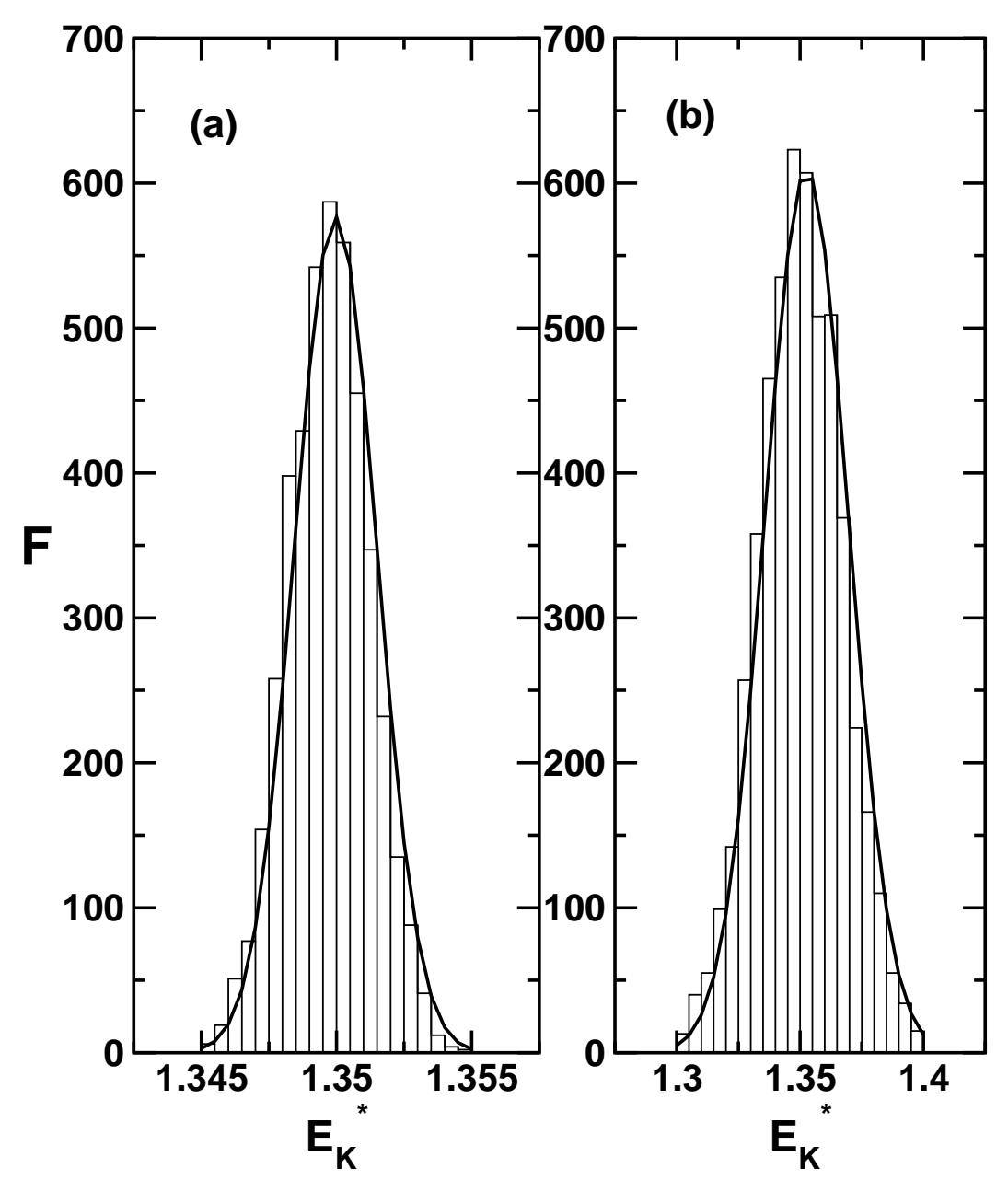

FIG. 1: Frequency versus reduced kinetic energy (Kinetic energy distribution) for a binary mixture with $\alpha=0.25$ and $N=4096$ particles at $T^{*}=0.9$. The vertical axis should be multiplied by a factor of $10^{3}$. (a) Results using an isokinetic thermostat and, (b) results applying a Nosé-Hoover thermostat. In each case the solid line represents the best fit to a Maxwell-Boltzmann distribution. 

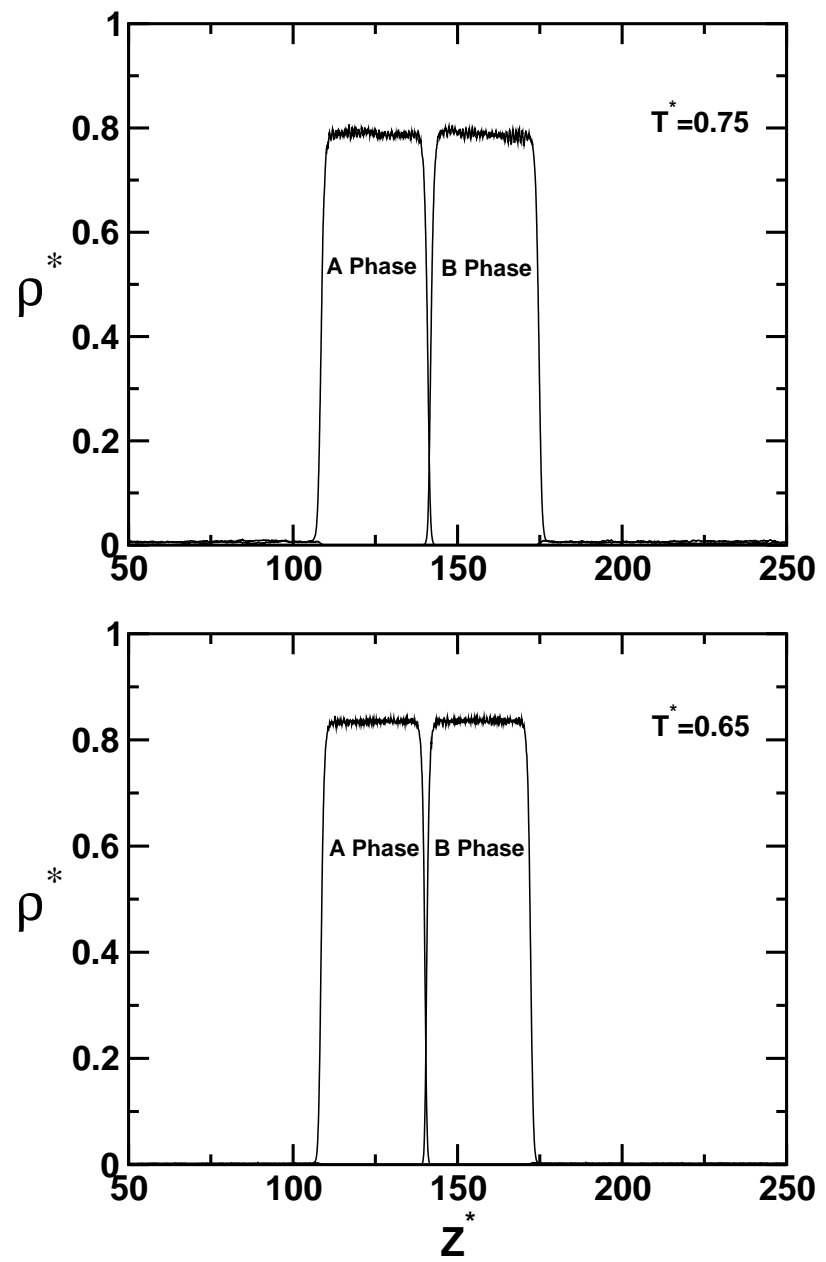

FIG. 2: Liquid-vapor-liquid reduced density profiles of the mixture for $\alpha=0.25$ and $N=4096$. Note that at these relatively low temperatures a liquid A-liquid B interface is formed. 

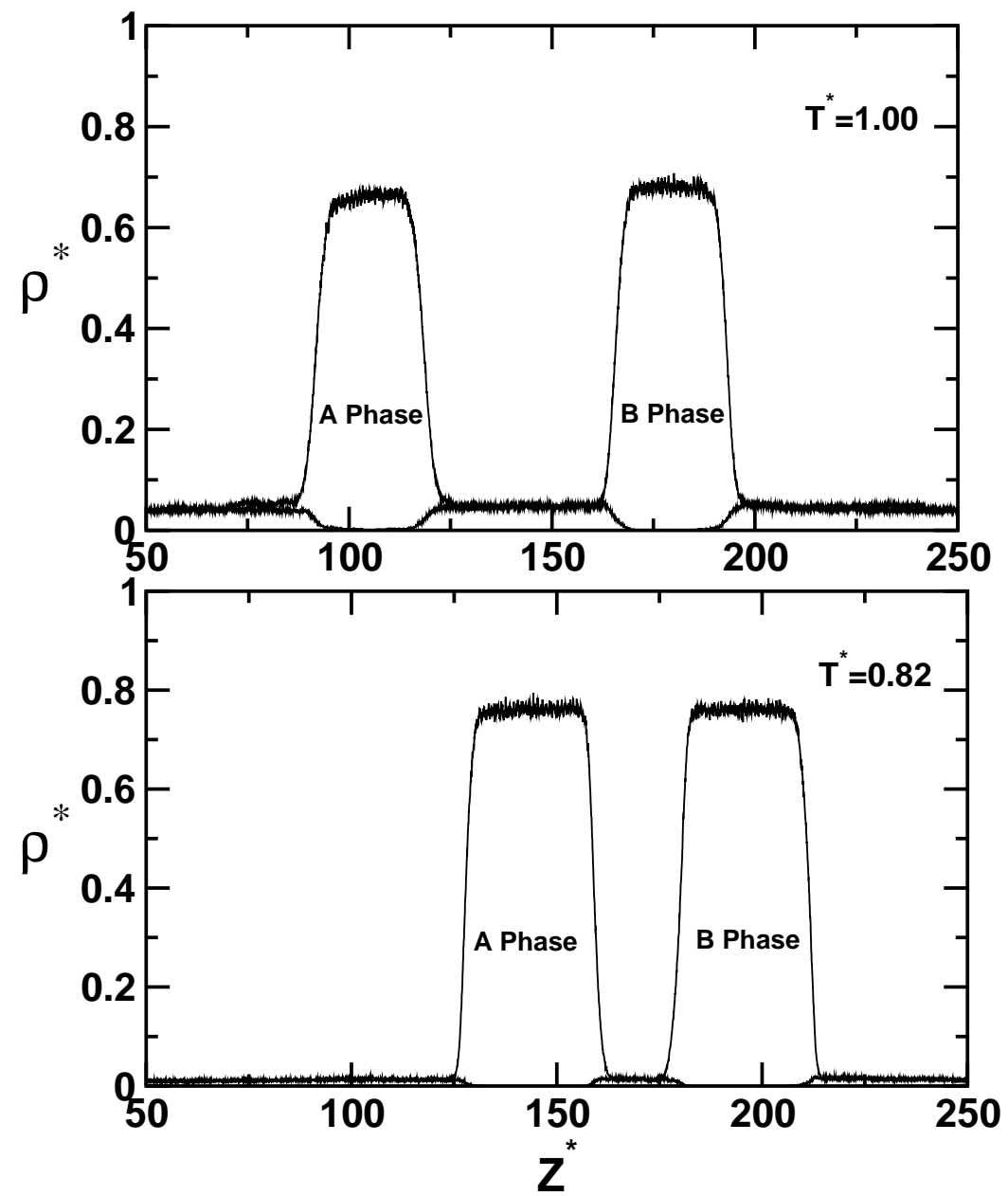

FIG. 3: Liquid-vapor-liquid reduced density profiles of the mixture for $\alpha=0.25$ and $N=4096$. At these higher temperatures the vapor phase spontaneously wets the liquid A-liquid-B phases. 

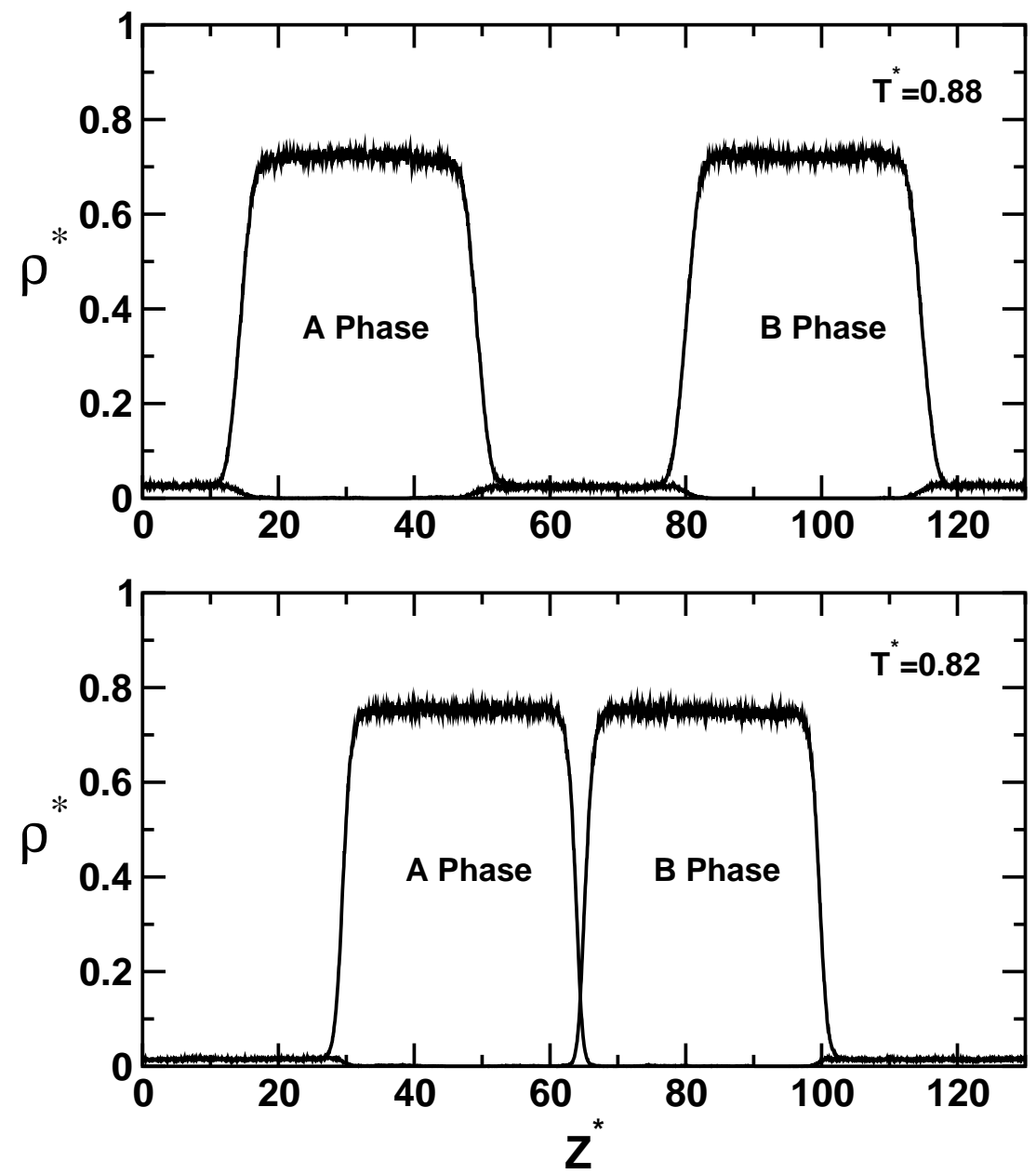

FIG. 4: Liquid-vapor-liquid reduced density profiles of the mixture for $\alpha=0.30$ and $N=4096$. At these higher temperatures the vapor spontaneously wets the liquid A-liquid-B phases. 


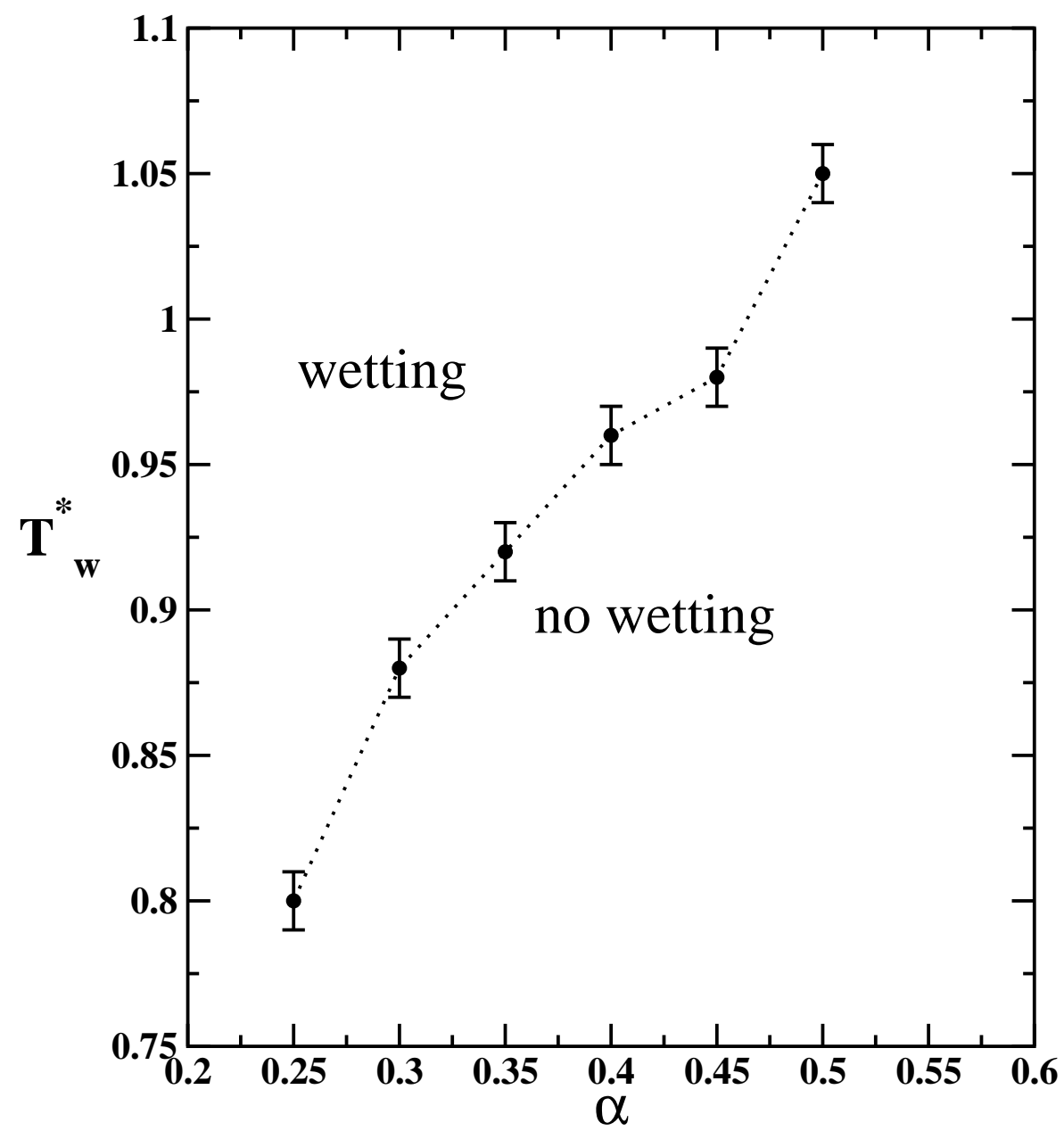

FIG. 5: Reduced wetting transition temperature as function of $\alpha$ for an equimolar binary mixture. These results were obtained from MD simulations with a $N=4096$ particles. The line is a guide to the eye. 


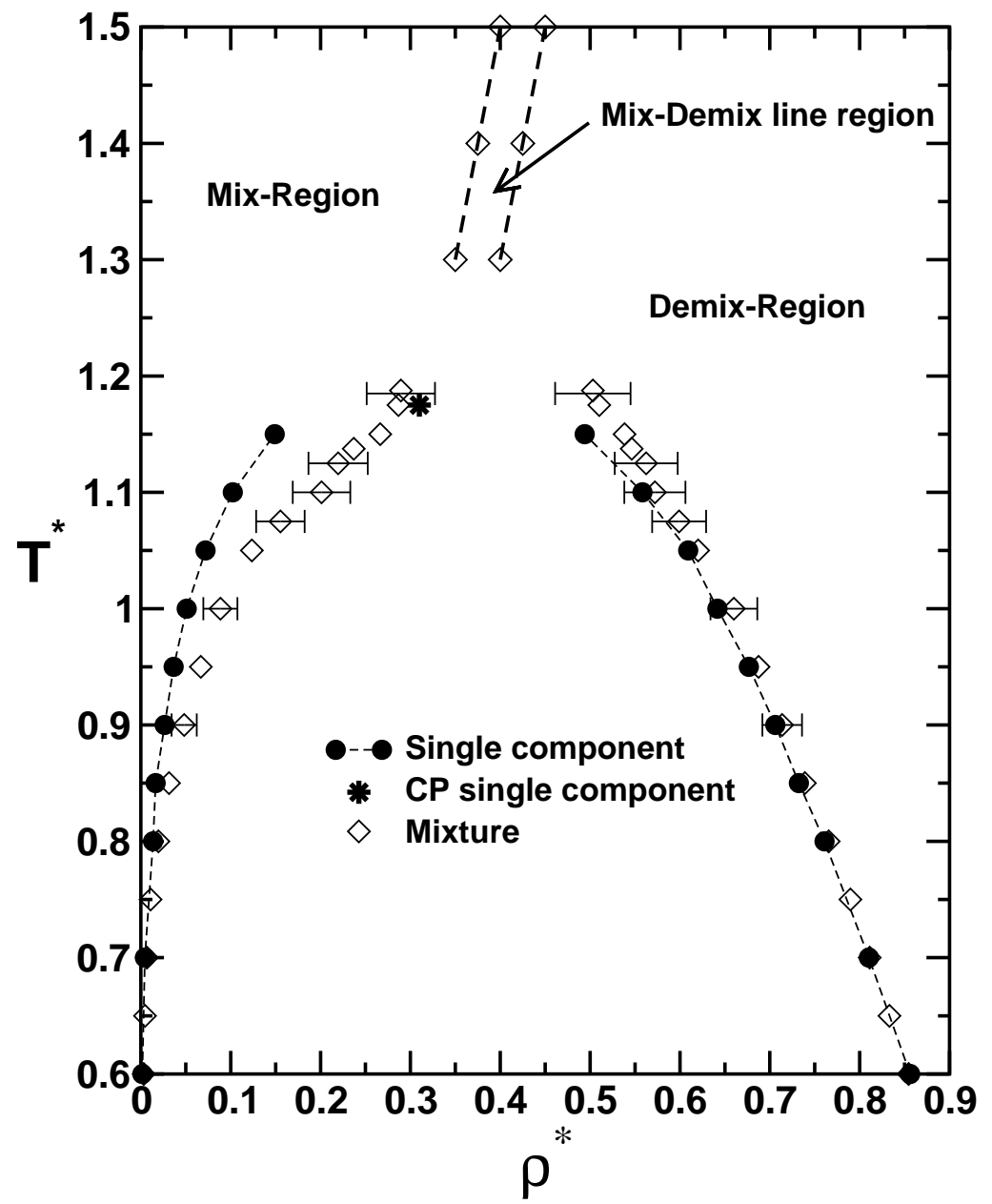

FIG. 6: $T^{*}$ versus $\rho^{*}$ phase diagram for a partially miscible mixture with $N=4096$ and $\alpha=0.25$. For comparison we have included the corresponding phase diagram of a single LJ fluid. 


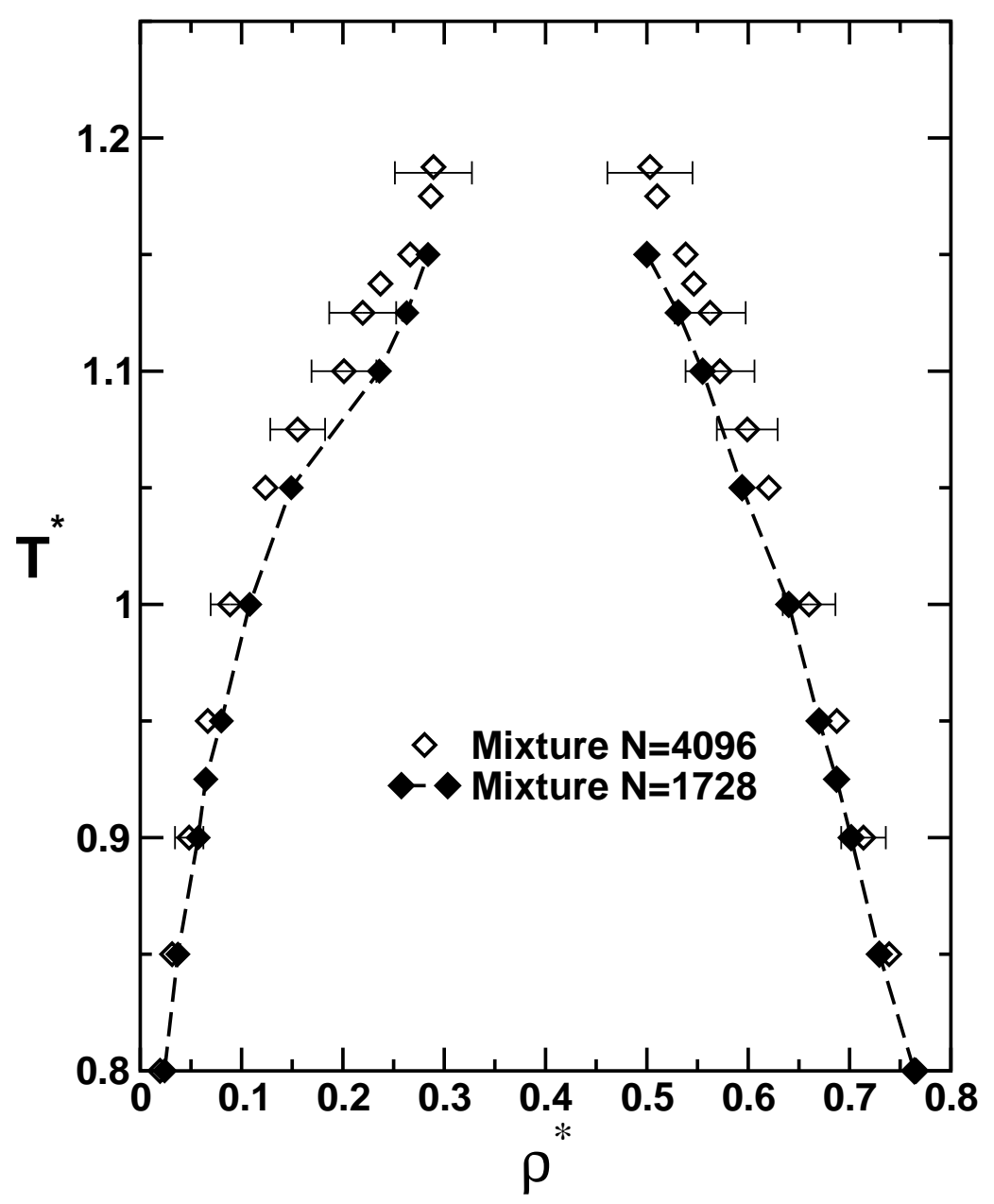

FIG. 7: $T^{*}$ versus $\rho^{*}$ phase diagram for a binary mixture with $N=1728(\diamond)$ and $N=4096(\diamond)$ particles and $\alpha=0.25$. 


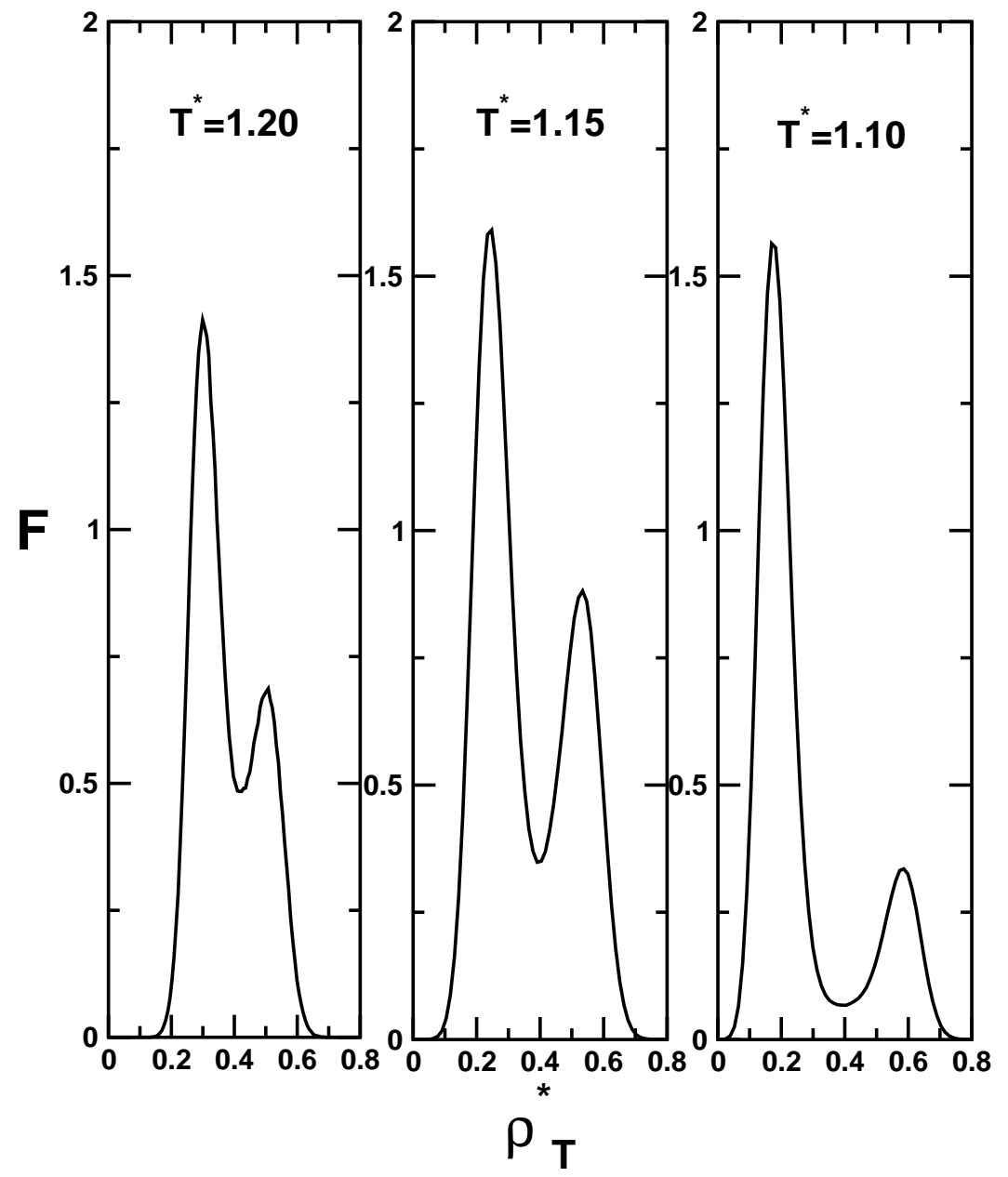

FIG. 8: Frequency versus total reduced density (total density distribution) of the mixture with $\alpha=0.25$ and $N=4096$ at $T^{*}=1.1,1.15$, and 1.2 . The vertical axis should be multiplied by a factor of $10^{3}$. These temperatures are slightly higher than the tricritical point temperature. 

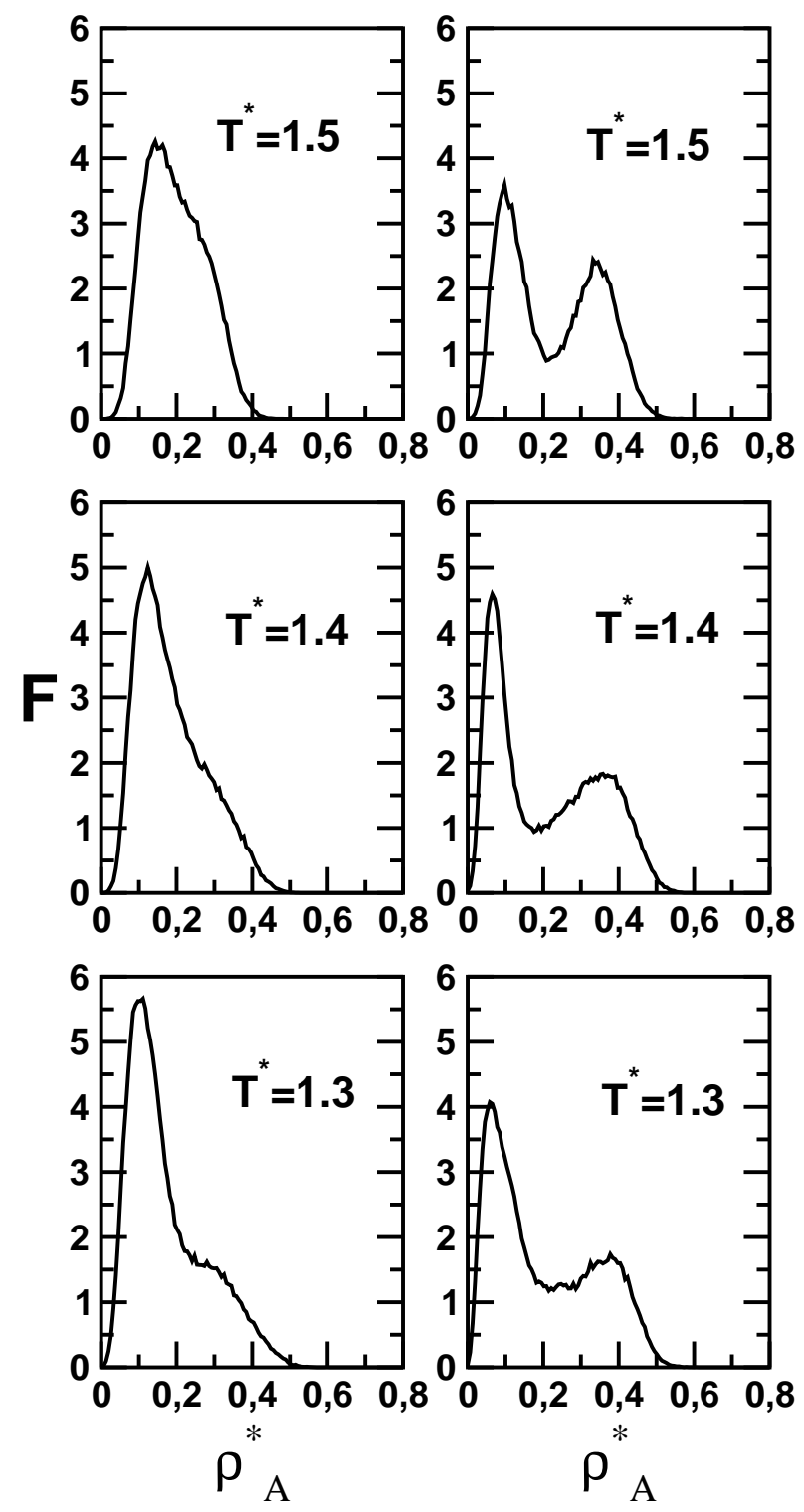

FIG. 9: Frequency versus reduced density (density distribution) for one of the fluid phases (fluid A) with $\alpha=0.25$ and $N=4096$. The vertical axis should be multiplied by a factor of $10^{3}$. The left columns, show the density distributions when the $\lambda$-line is approached form the mixing region at $T^{*}=1.3,1.4$, and 1.5. Note that they show a one peak structure. However, if the $\lambda$-line is approached from the demixing region at $T^{*}=1.3,1.4$, and 1.5 the density distributions, right columns, show a two peak structure. 


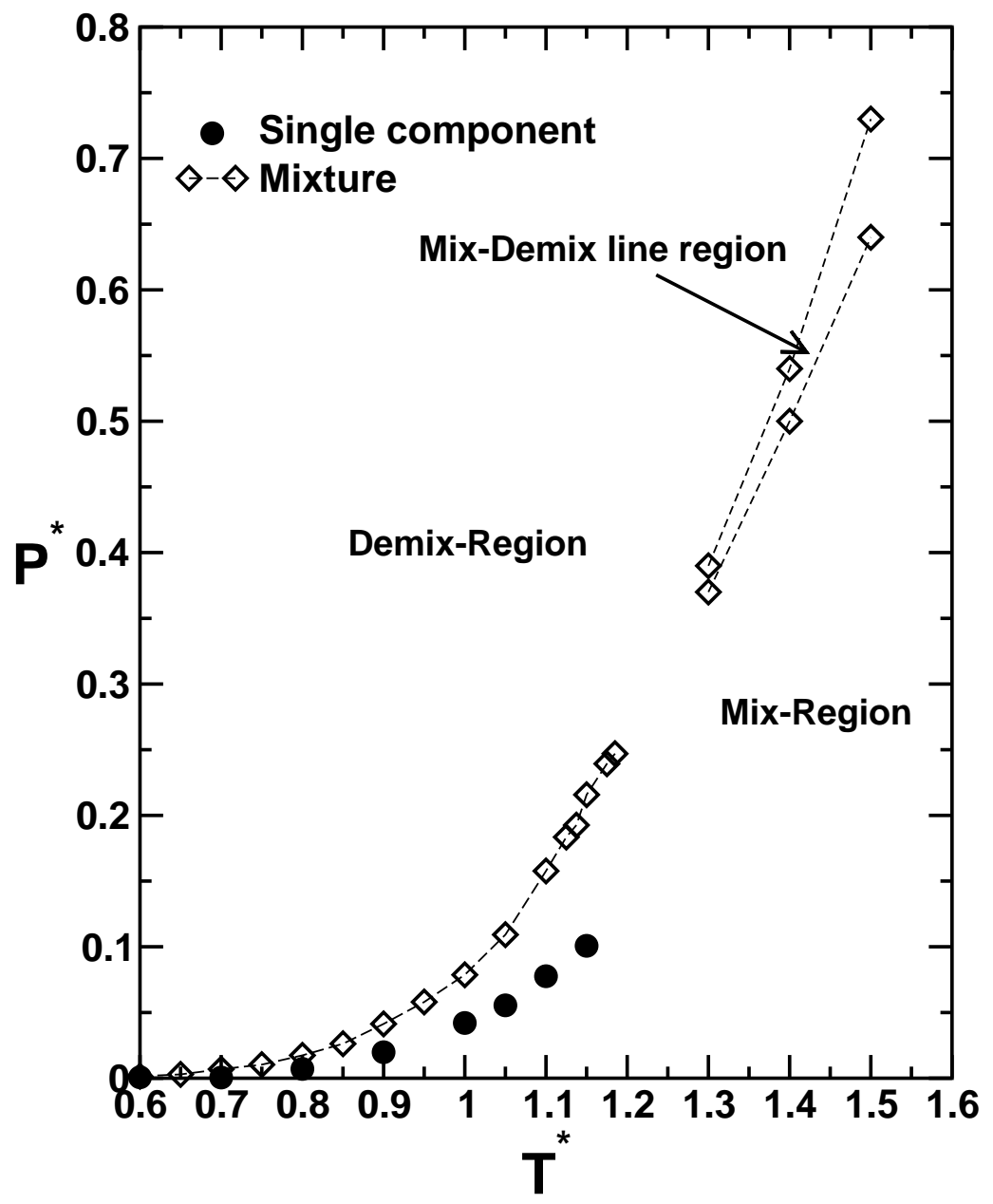

FIG. 10: Reduced pressure versus reduced temperature phase diagram for $\alpha=0.25$. 


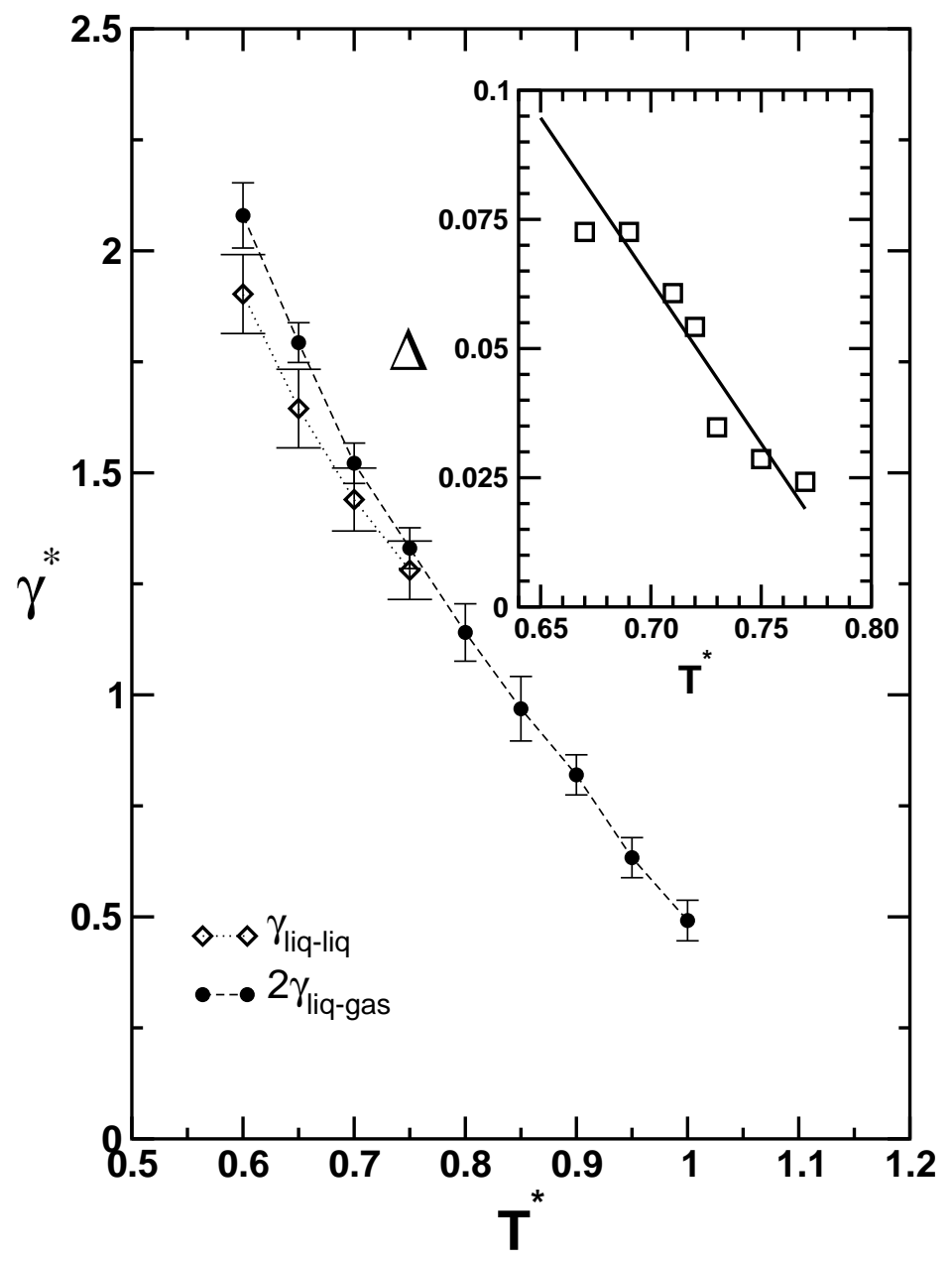

FIG. 11: Reduced interfacial tension as function of reduced temperature for both, LL and LV interfaces. In the inset we plot the difference $\Delta=2 \gamma_{\mathrm{LV}}-\gamma_{\mathrm{LL}}$ as a function of temperature. These results are representative of a mixture with $\alpha=0.25$. 


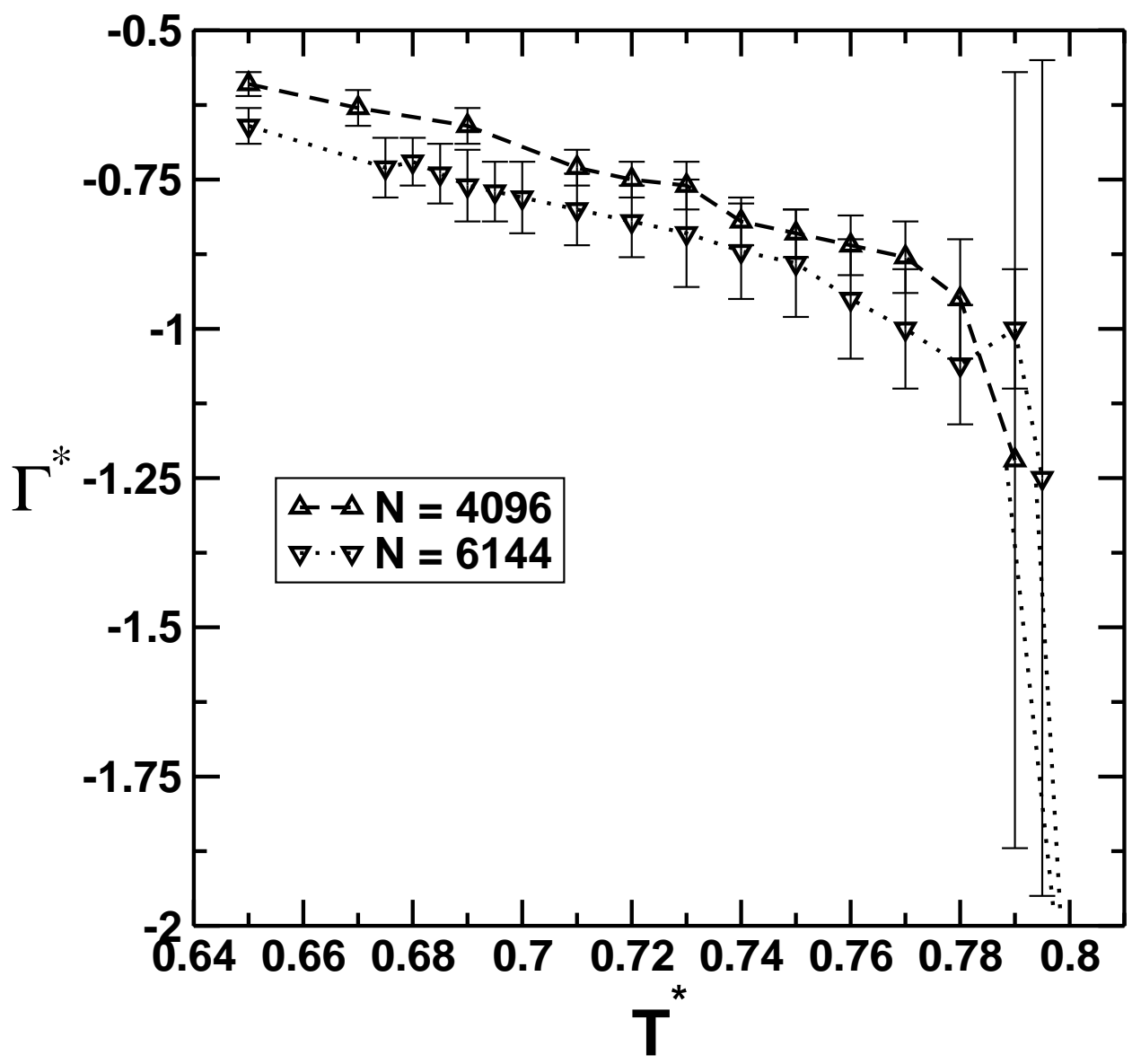

FIG. 12: Reduced adsorption as function of reduced temperature at the LL interface for two systems, $\mathrm{N}=4096(\triangle), \mathrm{N}=6144(\nabla)$ and $\alpha=0.25$. The two points that are closer to the wetting transition temperature show relatively large error bars. This is due to the increase of fluctuations of the interface width close to $T_{\mathrm{W}}^{*}(\alpha)$. In the present case, the vapor wets the LL interface at, $T_{\mathrm{W}}^{*}(\alpha)=0.80$, and the adsorption jumps from a finite negative value up to minus infinite. Dashed lines are a guide to the eye. 